\title{
Artificial Intelligence Applications in Type 2 Diabetes Mellitus Care: Focus on Machine Learning Methods
}

\author{
Shahabeddin Abhari ${ }^{1}$, Sharareh R. Niakan Kalhori ${ }^{1}$, Mehdi Ebrahimi ${ }^{2,3}$, Hajar Hasannejadasl ${ }^{1}$, \\ Ali Garavand ${ }^{4}$ \\ 'Department of Health Information Management, School of Allied Medical Sciences, Tehran University of Medical Sciences, Tehran, Iran \\ ${ }^{2}$ Department of Internal Medicine, School of Medicine, Tehran University of Medical Sciences, Tehran, Iran \\ ${ }^{3}$ Endocrinology and Metabolism Research Center, Endocrinology and Metabolism Research Institute, Tehran University of Medical Sciences, Tehran, Iran \\ ${ }^{4}$ Department of Health Information Management and Technology, School of Allied Medical Sciences, Shahid Beheshti University of Medical Sciences, Tehran, Iran
}

Objectives: The incidence of type 2 diabetes mellitus has increased significantly in recent years. With the development of artificial intelligence applications in healthcare, they are used for diagnosis, therapeutic decision making, and outcome prediction, especially in type 2 diabetes mellitus. This study aimed to identify the artificial intelligence (AI) applications for type 2 diabetes mellitus care. Methods: This is a review conducted in 2018. We searched the PubMed, Web of Science, and Embase scientific databases, based on a combination of related mesh terms. The article selection process was based on Preferred Reporting Items for Systematic Reviews and Meta-Analyses (PRISMA). Finally, 31 articles were selected after inclusion and exclusion criteria were applied. Data gathering was done by using a data extraction form. Data were summarized and reported based on the study objectives. Results: The main applications of AI for type 2 diabetes mellitus care were screening and diagnosis in different stages. Among all of the reviewed AI methods, machine learning methods with $71 \%(\mathrm{n}=22)$ were the most commonly applied techniques. Many applications were in multi method forms (23\%). Among the machine learning algorithms applications, support vector machine (21\%) and naive Bayesian (19\%) were the most commonly used methods. The most important variables that were used in the selected studies were body mass index, fasting blood sugar, blood pressure, HbAlc, triglycerides, low-density lipoprotein, high-density lipoprotein, and demographic variables. Conclusions: It is recommended to select optimal algorithms by testing various techniques. Support vector machine and naive Bayesian might achieve better performance than other applications due to the type of variables and targets in diabetes-related outcomes classification.

Keywords: Artificial Intelligence, Diabetes Mellitus, Machine Learning, Diabetes Care, Health Informatics

Submitted: May 14, 2019

Revised: 1st, July 15, 2019; 2nd, October 6, 2019

Accepted: October 9, 2019

\section{Corresponding Author}

Sharareh R. Niakan Kalhori

Department of Health Information Management, School of Allied Medical Sciences, Tehran University of Medical Sciences, Tehran, Iran. Tel: +98-21-88983025, E-mail: sh-rniakank@sina.tums.ac.ir (https://orcid.org/0000-0002-7577-1200)

This is an Open Access article distributed under the terms of the Creative Commons Attribution Non-Commercial License (http://creativecommons.org/licenses/by$\mathrm{nc} / 4.0 /$ ) which permits unrestricted non-commercial use, distribution, and reproduction in any medium, provided the original work is properly cited.

(c) 2019 The Korean Society of Medical Informatics

\section{Introduction}

Diabetes mellitus (DM) is known as the most prevalent metabolic disorder caused by the inability of the pancreas to produce insulin sufficiently or the body's lack of ability to use insulin effectively. There are various factors that cause diabetes, such as diet, lifestyle, and genetics [1-6]. Despite healthcare advances, the prevalence of diabetes is still growing, and currently, more than 200 million people worldwide are affected by DM [7-9]. The number of patients with diabetes, estimated by the World Health Organization (WHO) in 2004, was expected to rise from 171 million in 2000 to 
366 million by 2030 [10-12]; however, the recent estimation by the International Diabetes Federation (IDF) indicates that the number of diabetic patients will increase to $552 \mathrm{mil}-$ lion by $2030[5,13,14]$. DM can cause many complications, such as damage to the heart, blood vessels, eyes, kidneys, and nerves [5,15]. As a serious health concern, DM has been declared a global epidemic by the WHO due to its rapidly increasing incidence. Nowadays, many multidisciplinary studies are being done to support the prevention and treatment of DM, and it has become a worldwide research priority $[5,16]$. Thus, the surveillance, prevention, and control of diabetes and its complications using two or more disciplines are becoming increasingly important $[3,5,17]$ As each discipline has its own potential and added value, the combination of them might be a solution for DM [18].

Artificial intelligence (AI) has been applied in various medical fields for many purposes. Actually, AI is defined as "a field of science and engineering concerned with the computational understanding of what is commonly called intelligent behavior with the creation of artifacts that exhibit such behavior" [19-23]. AI algorithms have the potential to deliver better care especially in combination with recent technologies [24]. Because modern medicine has faced challenges regarding a large amount of data acquisition, analysis, and the application of the obtained knowledge to solving complex clinical problems, it is necessary to use AI capabilities for these purposes $[19,21,25]$. AI is composed of various intelligent algorithms and techniques, such as machine learning (ML), natural language processing (NLP), robotics, fuzzy logic (FL), expert systems (ES), knowledge base (KB), and the mix of two or more methods (multi- methods) [21,23]. ML methods are common with the ability of conducting these two tasks including either prediction (when the outcome variable is a value) or classification (when the outcome variable is a class) [26]. These tasks may also be applied in DM care for disease probability prediction, screening, diagnosis, treatment guidance, and complication management $[19,21,27,28]$. Several ML methods as a subcategory of AI have been applied to fulfill these clinical purposes using their own specific capabilities [29,30]. In detail, they include many techniques, such as artificial neural networks (ANN), support vector machine (SVM), decision tree (DT), and naive Bayes (NB), for prediction or classification of clinical outcomes. In routine medical practice, AI in medicine has been linked to the development of programs to help physicians in formulating diagnoses, making therapeutic decisions, and predicting the critical status, such as an emergency or worsening of a patient's condition, in their everyday duties $[17,22,24,29]$. Considering the importance of type 2 diabetes mellitus (T2DM) care as well as assuming that $\mathrm{AI}$ applications for diabetes care are effective tools, also due to a lack of studies to investigate the application of AI for T2DM care, this study reviewed AI algorithms and techniques for T2DM care with a specific focus on machine learning methods.

\section{Methods}

\section{Research Question}

This study aimed to identify AI applications in T2DM care. We reviewed papers that have reported the methods and techniques of AI in T2DM care. The study population com-

Table 1. Search strategy of the research

\section{Search strategy}

Database PubMed, Embase, ISI Web of Science (September 10, 2012 to September 10, 2017)

Limits Language (only resources in English), Species (studies on humans)

Data September 10, 2017 to October 10, 2017

\#1 “Diabetes Mellitus, Adult-Onset” OR “Diabetes Mellitus, Ketosis-Resistant” OR “Diabetes Mellitus, Maturity-Onset” OR “Diabetes Mellitus, Non-Insulin Dependent” OR “Diabetes Mellitus, Non-Insulin-Dependent” OR “Diabetes Mellitus, Noninsulin Dependent” OR “Diabetes Mellitus, Noninsulin-Dependent Diabetes Mellitus, Slow-Onset” OR “Diabetes Mellitus, Stable” OR “Diabetes Mellitus, Type II” OR “MODY” OR “Maturity-Onset Diabetes” OR “Maturity-Onset Diabetes Mellitus” OR “NIDDM” OR “Noninsulin-Dependent Diabetes Mellitus” OR “Type 2 Diabetes” OR “Type 2 Diabetes Mellitus”

\#2 “Artificial Intelligence” OR “Computer Heuristics” OR “Expert Systems” OR "Fuzzy Logic” OR "Knowledge Bases” OR "Machine Learning” OR "Natural Language Processing” OR "Neural Networks" OR "Robotics” OR "Prediction Model" 
prised people with T2DM, the intervention was using AI for health-related applications in the area of T2DM; the comparison was excluded; outcomes were the identification of various methods and techniques using AI in T2DM care.

\section{1) Search strategy}

This study was a review that was conducted in 2018. Searches were done in scientific databases, including PubMed, Web of Science, and Embase, based on the combination of related keywords based on mesh terms (Table 1). All steps of searches were done based on the Preferred Reporting Items for Systematic Reviews and Meta-Analyses (PRISMA) checklist. The searches were done by two researchers independently to prevent possible bias. The search results referred to a third person who reviewed contradictions, and made decisions in cases where there were disagreements.

The searches were limited to papers published in the English language and a 5-year period of time (2012-2017).

\section{2) Inclusion and exclusion criteria}

The inclusion criteria were original articles, clinical trials, and meta-analysis reports. Also, those works that had model performance evaluation, such as accuracy, sensitivity, specificity, and area under the curve (AUC) were included. Exclusion criteria were non-English articles, unavailable full text, other study types except clinical trial, and meta-analysis. Other article types included review articles, letters to editors, short communications, etc. Also conference articles were excluded. Non-human-study papers and papers without model performance evaluation criteria were removed.

\section{3) Selection process}

The process of article selection was based on PRISMA (Figure 1). Finally, 31 papers were selected. All steps in the selection and evaluation of the quality of the papers were done by two researchers. Cases of disagreement were referred to a third person to make the final decision. The data extraction form had eight categories, including the author's name, publication year, AI methods applied, algorithms used, health applications for T2DM, clinical variables, optimal algorithms, and the best performance of the model based on the related indexes. After data extraction, we summarized and reported the findings in tables and figures according to the objectives of the study.
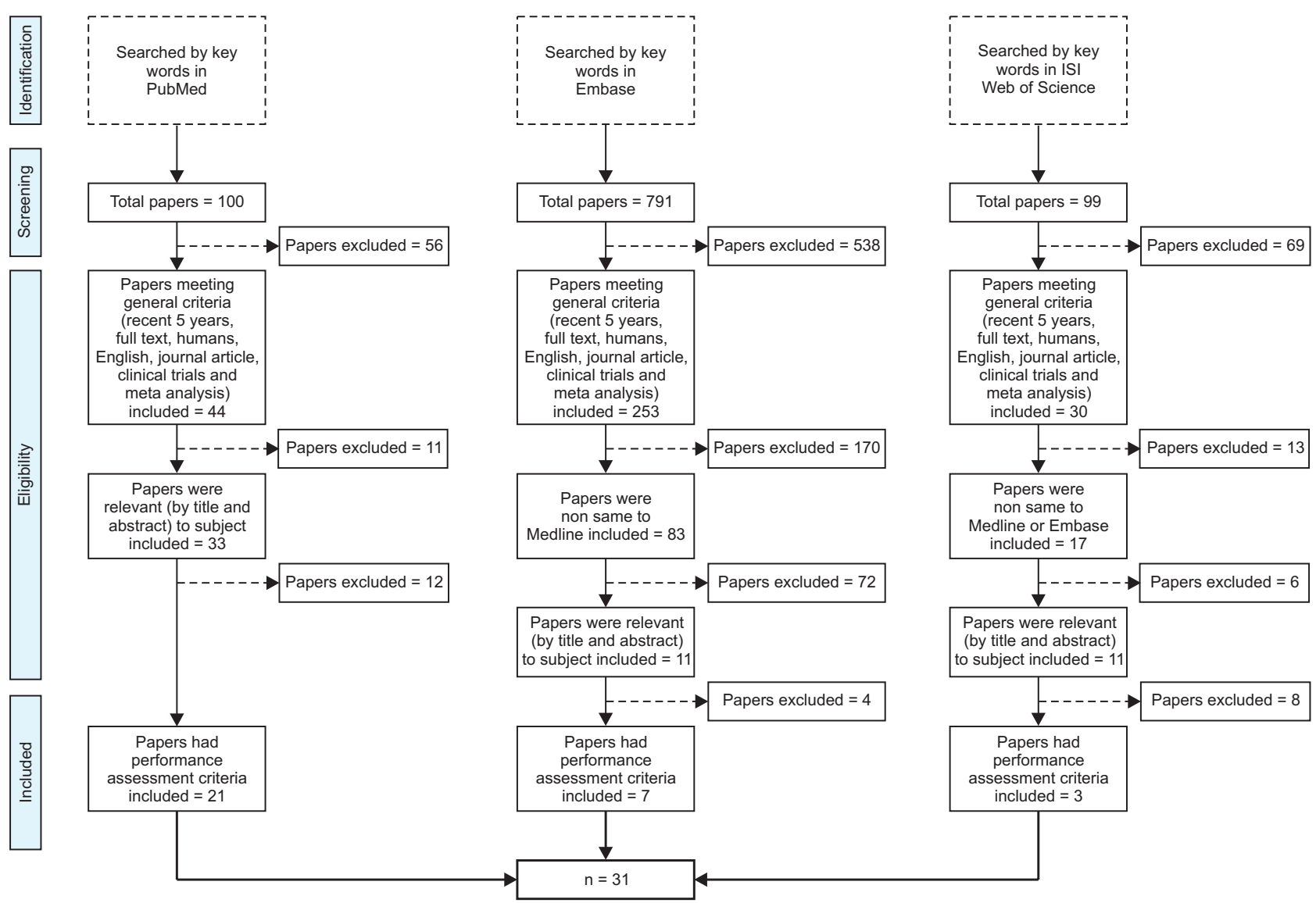

Figure 1. Process of PRISMA for data collection. 


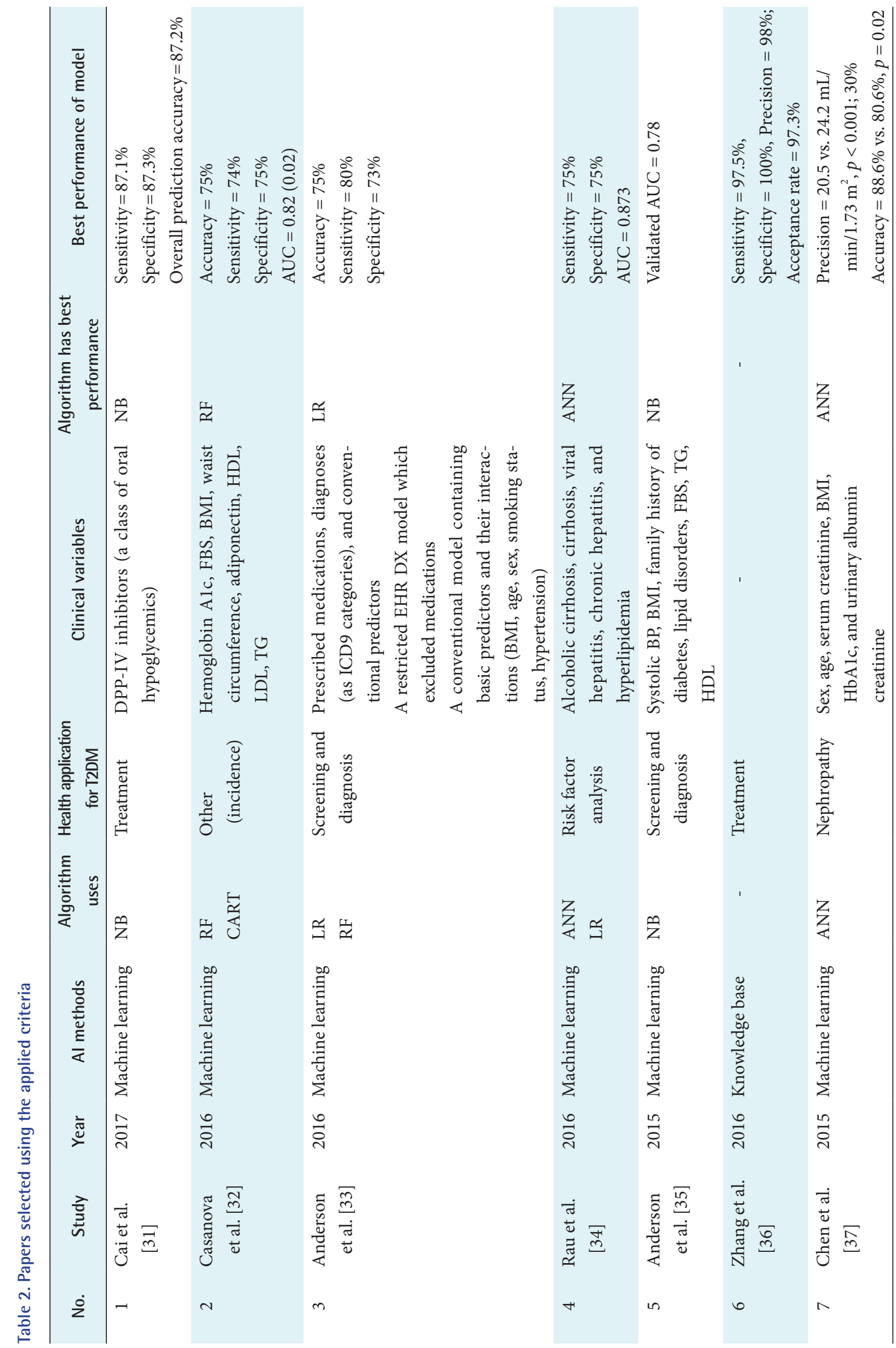




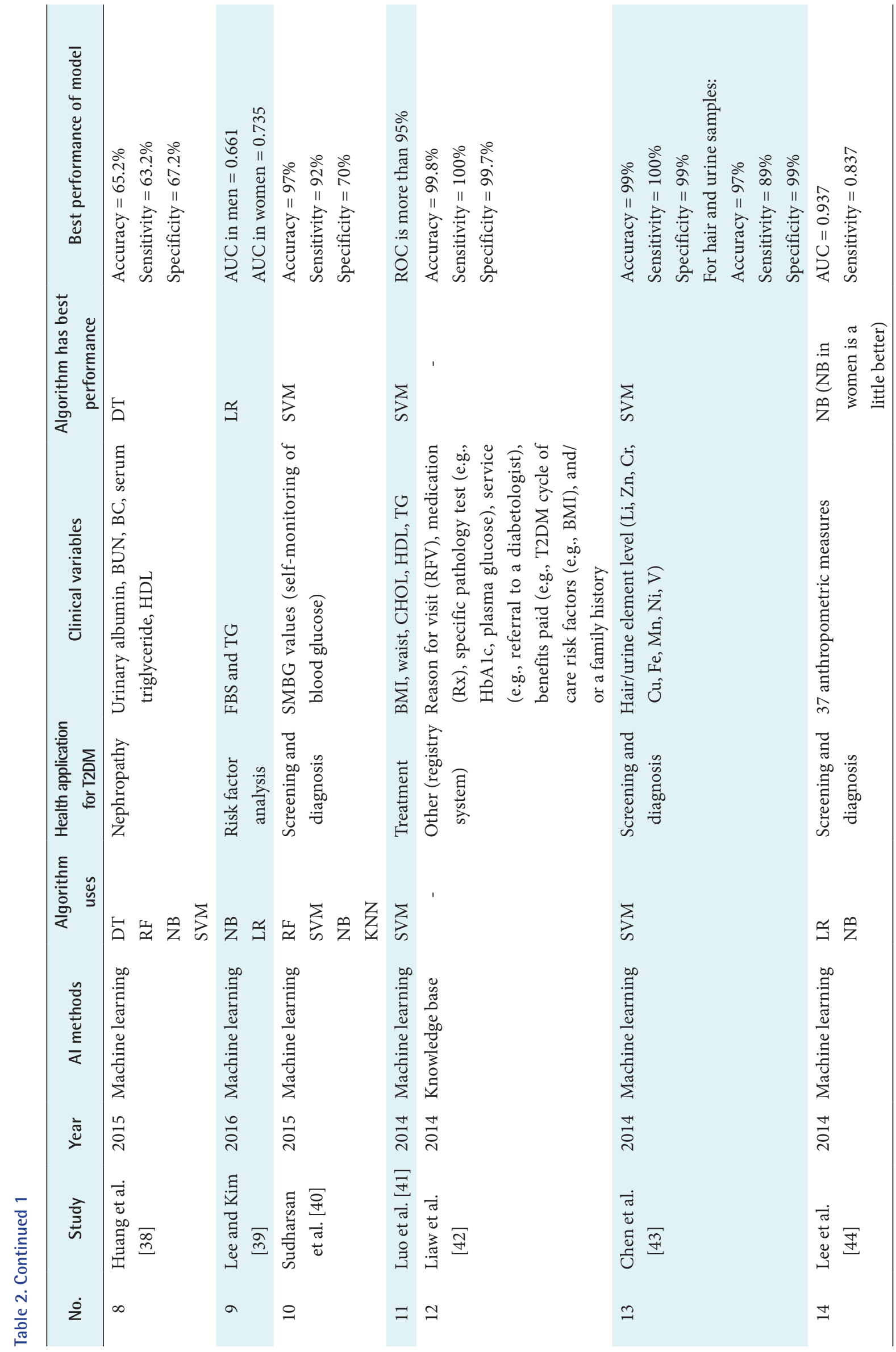




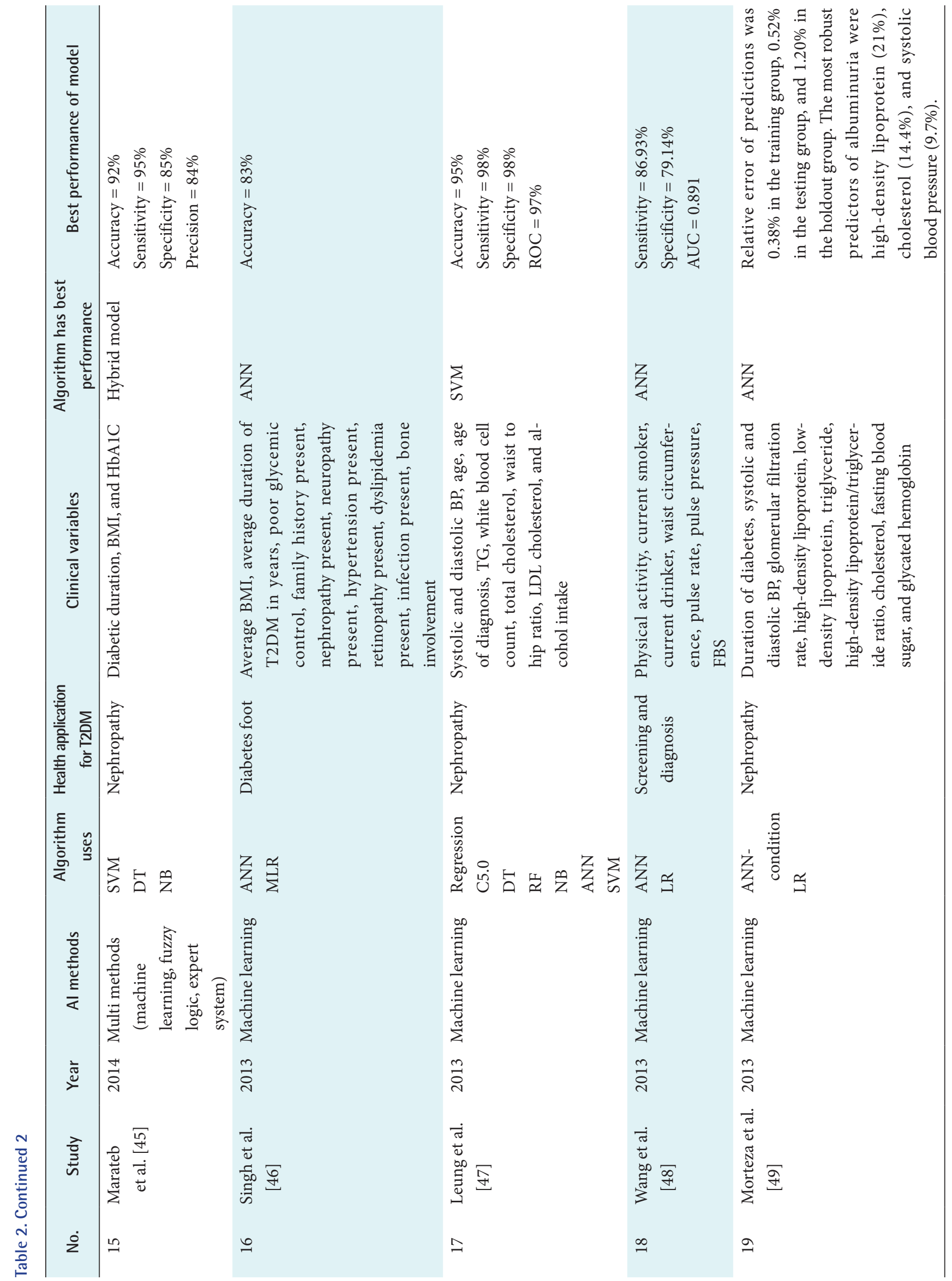




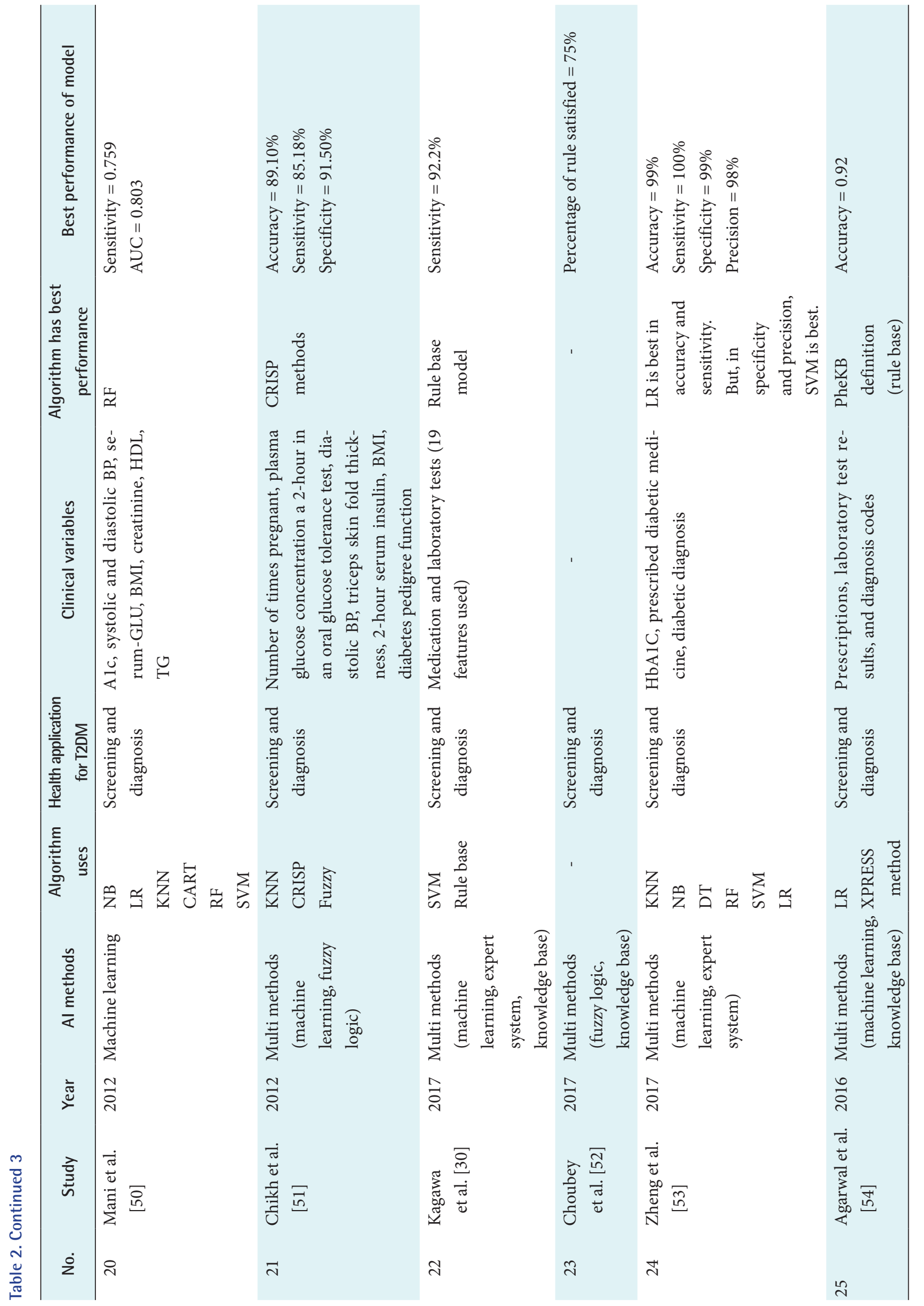




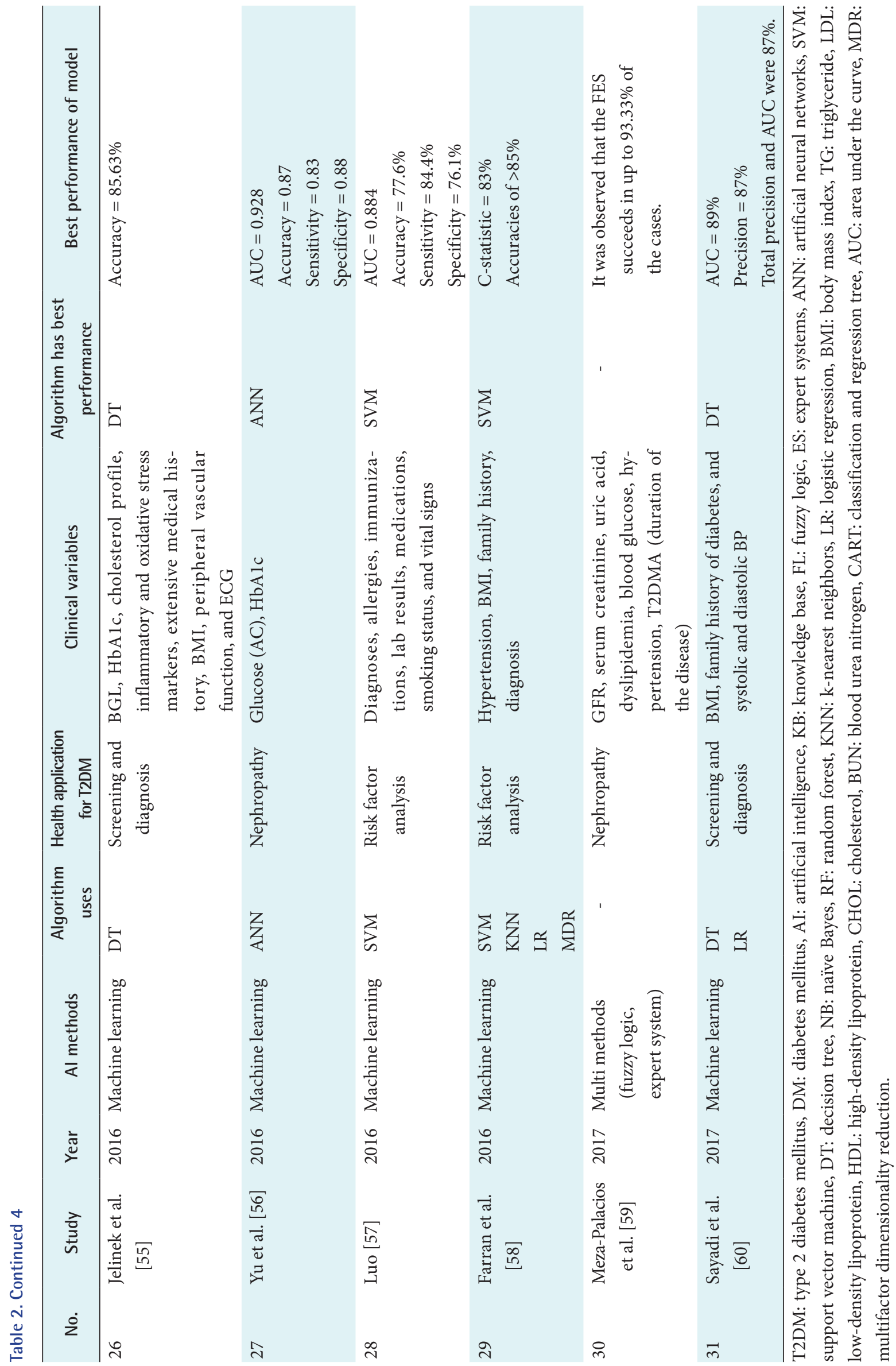




\section{Results}

According to the study search terms, 31 papers were reviewed in details. Table 2 showed the breakdown of articles categories. Most of the papers $(n=9)$ were published in 2016. Table 2 presents information about the selected studies.

In this study a variety of variables were considered, as shown in Table 2. They comprised the following: (1) AI methods (ML, KB, ANN, FL, ES, NLP, and robotic); (2) in case that the applied AI method was ML, we analyzed the algorithms of ML (ANN, SVM, NB, etc.); (3) we also studied the AI applications of health aspects for T2DM (risk factor analysis, screening and diagnosis, treatment, nephropathy, neuropathy, diabetic foot, etc.); (4) clinical variables were clinical features which were applied to be analyzed by using AI methods; and (5) the best performance values of mathematics models were listed and compared. For this purpose, we only studied those papers with reported performance

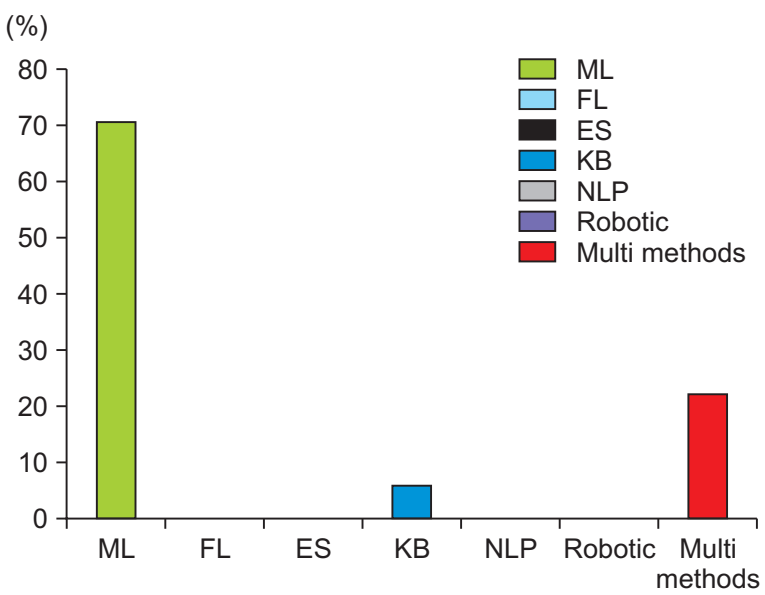

Figure 2. Frequency (percentage) of artificial intelligence methods used in type 2 diabetes mellitus. ML: machine learning, FL: fuzzy logic, ES: expert system, KB: knowledge base, NLP: natural language processing.

Table 3. Frequency of Al methods when multiple methods were applied

\begin{tabular}{lc}
\hline Al methods uses in multi methods & Frequency (\%) \\
\hline Machine learning & $5(31.25)$ \\
Fuzzy logic & $4(25.00)$ \\
Expert systems & $4(25.00)$ \\
Knowledge base systems & $3(18.75)$ \\
Total & $16(100)$ \\
\hline
\end{tabular}

AI: artificial intelligence. values as inclusion criteria.

As shown in Figure 2, 71\% of papers applied ML, 23\% used multiple methods, and just $6 \%$ of them used the KB method. Results show other methods, such as FL, ES, NLP and robotic, have not been applied to T2DM separately.

Table 3 shows the frequency of AI methods used when multiple methods were used. In 16 articles, two or more AI methods were used for T2DM concurrently. ML $(n=5)$ was the most frequently used method together with other AI techniques.

Figure 3 shows the frequency of algorithms applied specifically in ML. In 51 cases, ML algorithms were used for T2DM care. As seen in Figure 3, SVM was the most frequently used algorithm. NB was the second most commonly used algorithm. Moreover, the results show that logistic regression, which is one of the most famous statistical algorithms was applied in 13 cases in the reviewed research.

Figure 4 shows the frequency of AI applications to health aspects of T2DM. The results showed that the most common

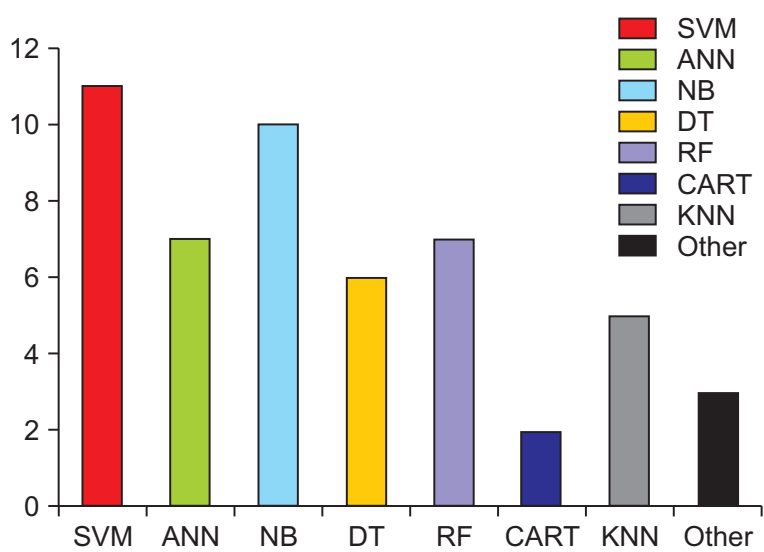

Figure 3. Frequency of machine learning algorithms used for type 2 diabetes mellitus care. SVM: support vector machine, ANN: artificial neural network, NB: naïve Bayes, DT: decision tree, RF: random forest, CART: classification and regression trees, KNN: k-nearest neighbor.

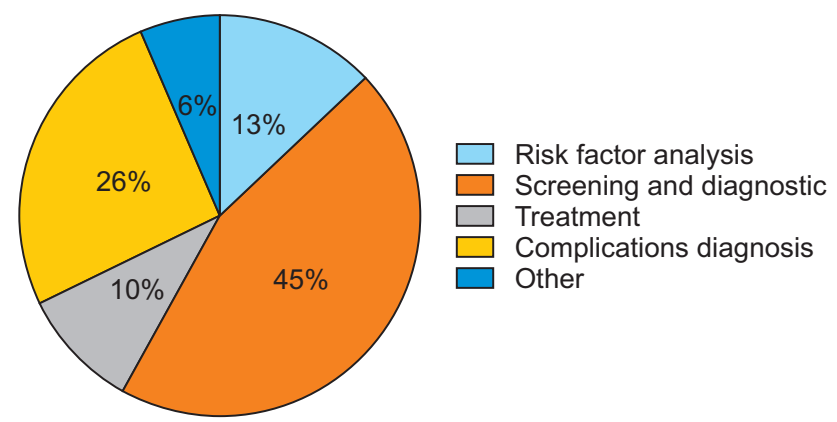

Figure 4. Frequency of artificial intelligence applications for health aspects of type 2 diabetes mellitus. 
medical application of AI for T2DM care was in screening and diagnosis, with a $45 \%$ frequency. Then, complication diagnosis was the next most frequent application of AI (26\%).

\section{Discussion}

According to the results, the main AI applications are related to ML use for knowledge production and model development, which has been widely used in many healthcare applications [9]. It is a key technology to transform biomedical datasets into actionable knowledge that is useful for the advancement of clinical practice and healthcare through rules developed by medical experts, statistical methods, and ML algorithms with the ability of self-improvement $[9,30,61]$. Actually, ML methods often achieve high accuracy due to looser assumptions regarding data distribution in comparison to other methods $[8,10,11,17,30]$. The results of this study indicate that main clinical variables in running and designing ML models and systems for type 2 diabetes care, were body mass index (BMI), fasting blood sugar (FBS), blood pressure (systolic and diastolic), HbAlc, triglycerides (TG), low-density lipoprotein (LDL), high-density lipoprotein (HDL), family history, and demographic variables (Table 2 ). This study supports the evidence from previous reports that have identified some important clinical variables that are used in data mining methods in diabetes research [29].

According to the results of this study, models based on ML algorithms in T2DM care have been mainly focused on pre-diabetes screening and diagnostic outcomes, risk factor analysis, treatment, and complication categorization. That is, ML algorithms have been mainly used to classify diabetic prone cases for pre-diabetes, diabetes, and advanced diabetes based on the patients' HbA1c level. They are used to analyze T2DM risk factors in various populations to determine which categories of patients may require more attention to prevent (1) disease occurrence, (2) progress to worse stages, and (3) advancing to complications [29,51]. This study revealed that there has been limited research on estimating the probability of these outcomes rather than classifying patients based on their disease outcomes in the given three categories. According to previous classification experiments, SVM, which has been widely used for diabetic data analysis has outperformed other algorithms. This may be attributed to its capability to apply hyperlinks to separate classes in a threedimensional space $[61,62]$. Also, NB, which has been used in several cases for the purpose of determining diabetes-related outcomes is usually applied for prediction task only for small datasets [29]. Using this algorithm, the probability of spe- cific outcome occurrence, such as pre-diabetes occurrence probability, can be calculated; while by using the algorithm of NB the probability of specific outcome occurrence such as pre-diabetes occurrence can be calculated, there is no report of conducting this work in practice. Generally, ANN, and DT in the frame of C5, CART, and RF have been applied for both classification and prediction purposes $[63,64]$; however, there has been no report of applying these algorithms for diabetes-related outcome prediction using big data, even with a satisfactory and generalization level.

The performance of AI techniques and its models applied to T2DM care are shown in the eighth and ninth columns of Table 2. Regarding the best performance of ML techniques, it is obvious that there are no particular best techniques for every condition. For example, decision trees that classify cases by sorting them based on feature values show varying performance in different studies. It has been reported that there is a relationship between the performance of applied techniques and the following subjects, including the type of issues analyzed, the type of input data (discrete or continuous), and finally, the emerging overlapping in outcome classes [65-67].

In the area of AI application for T2DM care, there have been some scattered observational studies which need more trials to be applied in routine care. For example, a study presented a patient-level sequential modeling approach to implementing personalized prescription. In this approach, previous records of a patient were applied to the prediction of future prescriptions to improve accuracy. The effectiveness of this method was tested by implementing prediction models based on recurrent neural networks (RNN) [68]. Another study performed a literature review of efforts to use artificial intelligence techniques for diabetes management. The results demonstrate that AI methods are not only suitable for use in clinical practice but also self-management of diabetes. Also, these methods have the potential for improving patients' quality of life [24]. The present study focused on ML techniques to predict T2DM outcomes. The frequencies of various types of applications, as well as health aspects of T2DM care, were studied. Other AI methods may provide additional powerful tools to support diabetes care.

Furthermore, an experiment used the Q-learning algorithm in the area of reinforcement learning of AI to develop personalized treatment plans based on glucose level to provide different basal dose levels automatically for the treatment of each diabetes patient. This approach is a model free of reinforcement learning technique, which is used to determine the optimal treatment policy from a patient's treatment 
history and related laboratory results. The results reveal that this approach is an effective tool for personalized diabetes management [69].

As seen in Table 2, there have been several reports of multiple-method applications in T2DM care. Most of the ML algorithms have been used with FL, KB, and ES to create multiple-method-based systems for users in the area of diabetes control, including patients, physicians/nurses, managers, and policymakers. In the frame of these systems, ML applications for T2DM data analysis are used for knowledge production to enrich the system's knowledge base incrementally. They use diabetes patients' real data collected in a data repository over time to create a rich knowledge base. The knowledge base might also include experts' knowledge. The fuzzy method can either use real data or experts' knowledge to enrich the knowledge base [70].

Similarly, robotics technologies learn from humans and from the environment [71]. Despite many applications of ML in the framework of an indirect ES or direct ES, according to our inclusion criteria, there have been no reports of studies using robotics and NLP technology to support T2DM care that have evaluated model performance at the same time.

There have been reports on other areas of DM research using robotics, such as using a robot for pancreas transplantation, pancreatectomy surgery, and monitoring and training to improve the care of elderly with dementia [72-74]. Also, the management of type 1 diabetes in children is improved by robots. Robots can keep track of individuals' performance and can offer tailored lessons to enhance learning [75].

One of the most interesting findings of this study was that AI has often shown success in the prediction of related issues in T2DM. For example, in treatment, a study predicted DPPIV inhibitors with ML approaches with $87.2 \%$ accuracy [31]. In screening and diagnosis, another investigation predicted hypoglycemia using ML models with 97\% accuracy [40]. In the diagnosis of complications (nephropathy), a study predicted microalbuminuria using multiple AI methods (ML, FL, ES) with $92 \%$ accuracy [45]. In risk factor analysis, another study predicted the risk of type 2 diabetes, hypertension, and comorbidity using ML models with $85 \%$ accuracy [58].

The results of this study may support researchers and developers of AI-based systems and models in the care of patients with T2DM in choosing methods, models, algorithms, and efficient and optimal systems. It is suggested that ML, specifically SVM and NB, algorithms are considered by designers and developers of patterns and systems. The present study also identified the most important clinical variables used in the design and development of artificial intelligence systems and models for the care of patients with T2DM. This can provide insights for choosing key variables in T2DM, and in data analysis and system development using AI-based methods.

One of the limitations of this study was the number of databases that were reviewed. In this work, PubMed, Embase, and Web of Science were reviewed. In fact, the focus of this review was clinical databases; therefore, more technical databases, such as IEEE and Scopus should be considered for further reviews. Future investigation should focus on the effect of AI on clinical outcomes and its impact.

\section{Conflict of Interest}

No potential conflict of interest relevant to this article was reported.

\section{ORCID}

Shahabeddin Abhari (http://orcid.org/0000-0001-6585-091X) Sharareh R. Niakan Kalhori (http://orcid.org/0000-0002-7577-1200)

Mehdi Ebrahimi (http://orcid.org/0000-0002-2783-1248)

Hajar Hasannejadasl (http://orcid.org/0000-0001-5262-7399)

Ali Garavand (http://orcid.org/0000-0003-2238-0510)

\section{References}

1. Danaei G, Finucane MM, Lu Y, Singh GM, Cowan MJ, Paciorek CJ, et al. National, regional, and global trends in fasting plasma glucose and diabetes prevalence since 1980: systematic analysis of health examination surveys and epidemiological studies with 370 country-years and 2.7 million participants. Lancet 2011;378(9785):31-40.

2. World Health Organization. Global status report on noncommunicable diseases 2010. Geneva, Switzerland: World Health Organization; 2011.

3. World Health Organization. Definition, diagnosis and classification of diabetes mellitus and its complications: report of a WHO consultation. Part 1: Diagnosis and classification of diabetes mellitus. Geneva, Switzerland: World Health Organization; 1999.

4. American Diabetes Association. Diagnosis and classification of diabetes mellitus. Diabetes Care 2014;37(Supplement 1):S81-S90.

5. World Health Organization. Global report on diabetes. Geneva, Switzerland: World Health Organization; 2016.

6. Robertson AR, Fernando B, Morrison Z, Kalra D, 
Sheikh A. Structuring and coding in health care records: a qualitative analysis using diabetes as a case study. J Innov Health Inform 2015;22(2):275-83.

7. Quinn L. Glucose monitoring in the acutely ill patient with diabetes mellitus. Crit Care Nurs Q 1998;21(3):8596.

8. Naqshbandi M, Harris SB, Esler JG, Antwi-Nsiah F. Global complication rates of type 2 diabetes in Indigenous peoples: A comprehensive review. Diabetes Res Clin Pract 2008;82(1):1-17.

9. Amos AF, McCarty DJ, Zimmet P. The rising global burden of diabetes and its complications: estimates and projections to the year 2010. Diabet Med 1997;14 Suppl 5:S1-85.

10. Vashist SK. Non-invasive glucose monitoring technology in diabetes management: a review. Anal Chim Acta 2012;750:16-27.

11. Zhang P, Zhang X, Brown J, Vistisen D, Sicree R, Shaw J, et al. Global healthcare expenditure on diabetes for 2010 and 2030. Diabetes Res Clin Pract 2010;87(3):293-301.

12. International Diabetes Association. New estimates for 2012 of diabetes prevalence, mortality, and healthcare expenditures. Brussels, Belgium: International Diabetes Association; 2012.

13. Wild S, Roglic G, Green A, Sicree R, King H. Global prevalence of diabetes: estimates for the year 2000 and projections for 2030. Diabetes Care 2004;27(5):1047-53.

14. Whiting DR, Guariguata L, Weil C, Shaw J. IDF diabetes atlas: global estimates of the prevalence of diabetes for 2011 and 2030. Diabetes Res Clin Pract 2011;94(3):31121.

15. Sautin M, Suchkov S. Opportunities of predictive medicine at the treatment of diabetes mellitus complications. EPMA J 2014;5(Suppl 1):A73.

16. Roberts CK, Sindhu KK. Oxidative stress and metabolic syndrome. Life Sci 2009;84(21-22):705-12.

17. Dagliati A, Marini S, Sacchi L, Cogni G, Teliti M, Tibollo $\mathrm{V}$, et al. Machine learning methods to predict diabetes complications. J Diabetes Sci Technol 2018;12(2):295302.

18. Dargis V, Pantelejeva O, Jonushaite A, Vileikyte L, Boulton AJ. Benefits of a multidisciplinary approach in the management of recurrent diabetic foot ulceration in Lithuania: a prospective study. Diabetes Care 1999;22:1428-31.

19. Hamet P, Tremblay J. Artificial intelligence in medicine. Metabolism 2017;69S:S36-S40.

20. Szolovits P. Artificial intelligence in medicine. Boulder
(CO): Westview Press; 1982.

21. Ramesh AN, Kambhampati C, Monson JR, Drew PJ. Artificial intelligence in medicine. Ann R Coll Surg Engl 2004;86(5):334-8.

22. Rigla M, Garcia-Saez G, Pons B, Hernando ME. Artificial intelligence methodologies and their application to diabetes. J Diabetes Sci Technol 2018;12(2):303-10.

23. Nilsson NJ. Artificial intelligence: a new synthesis. San Francisco (CA): Morgan Kaufmann; 1998.

24. Contreras I, Vehi J. Artificial intelligence for diabetes management and decision support: literature review. J Med Internet Res 2018;20(5):e10775.

25. Fallah M, Niakan Kalhori SR. Systematic review of data mining applications in patient-centered mobilebased information systems. Healthc Inform Res 2017;23(4):262-70.

26. Han J, Pei J, Kamber M. Data mining: concepts and techniques. Amsterdam, Netherlands: Elsevier; 2011.

27. Patel VL, Shortliffe EH, Stefanelli M, Szolovits P, Berthold MR, Bellazzi R, et al. The coming of age of artificial intelligence in medicine. Artif Intell Med 2009;46(1):5-17.

28. Schwartz WB, Patil RS, Szolovits P. Artificial intelligence in medicine. N Engl J Med 1987;316(11):685-8.

29. Kavakiotis I, Tsave O, Salifoglou A, Maglaveras N, Vlahavas I, Chouvarda I. Machine learning and data mining methods in diabetes research. Comput Struct Biotechnol J 2017;15:104-16.

30. Kagawa R, Kawazoe Y, Ida Y, Shinohara E, Tanaka K, Imai $\mathrm{T}$, et al. Development of type 2 diabetes mellitus phenotyping framework using expert knowledge and machine learning approach. J Diabetes Sci Technol 2017;11(4):791-9.

31. Cai J, Li C, Liu Z, Du J, Ye J, Gu Q, et al. Predicting DPP-IV inhibitors with machine learning approaches. J Comput Aided Mol Des 2017;31(4):393-402.

32. Casanova R, Saldana S, Simpson SL, Lacy ME, Subauste AR, Blackshear C, et al. Prediction of incident diabetes in the Jackson Heart Study using high-dimensional machine learning. PLoS One 2016;11(10):e0163942.

33. Anderson AE, Kerr WT, Thames A, Li T, Xiao J, Cohen MS. Electronic health record phenotyping improves detection and screening of type 2 diabetes in the general United States population: a cross-sectional, unselected, retrospective study. J Biomed Inform 2016;60:162-8.

34. Rau HH, Hsu CY, Lin YA, Atique S, Fuad A, Wei LM, et al. Development of a web-based liver cancer prediction model for type II diabetes patients by using an artificial 
neural network. Comput Methods Programs Biomed 2016;125:58-65.

35. Anderson JP, Parikh JR, Shenfeld DK, Ivanov V, Marks C, Church BW, et al. Reverse engineering and evaluation of prediction models for progression to type 2 diabetes: an application of machine learning using electronic health records. J Diabetes Sci Technol 2015;10(1):6-18.

36. Zhang YF, Tian Y, Zhou TS, Araki K, Li JS. Integrating HL7 RIM and ontology for unified knowledge and data representation in clinical decision support systems. Comput Methods Programs Biomed 2016;123:94-108.

37. Chen J, Tang H, Huang H, Lv L, Wang Y, Liu X, et al. Development and validation of new glomerular filtration rate predicting models for Chinese patients with type 2 diabetes. J Transl Med 2015;13:317.

38. Huang GM, Huang KY, Lee TY, Weng J. An interpretable rule-based diagnostic classification of diabetic nephropathy among type 2 diabetes patients. BMC Bioinformatics 2015;16 Suppl 1:S5.

39. Lee BJ, Kim JY. Identification of type 2 diabetes risk factors using phenotypes consisting of anthropometry and triglycerides based on machine learning. IEEE J Biomed Health Inform 2016;20(1):39-46.

40. Sudharsan B, Peeples M, Shomali M. Hypoglycemia prediction using machine learning models for patients with type 2 diabetes. J Diabetes Sci Technol 2015;9(1):86-90.

41. Luo S, Chen S, Pan L, Zhang T, Han L, Wang Y, et al. Exploring the effects of intervention for those at high risk of developing type 2 diabetes using a computer simulation. Comput Biol Med 2014;53:105-14.

42. Liaw ST, Taggart J, Yu H, de Lusignan S, Kuziemsky C, Hayen A. Integrating electronic health record information to support integrated care: practical application of ontologies to improve the accuracy of diabetes disease registers. J Biomed Inform 2014;52:364-72.

43. Chen $\mathrm{H}$, Tan $\mathrm{C}$, Lin $\mathrm{Z}$, Wu T. The diagnostics of diabetes mellitus based on ensemble modeling and hair/urine element level analysis. Comput Biol Med 2014;50:70-5.

44. Lee BJ, Ku B, Nam J, Pham DD, Kim JY. Prediction of fasting plasma glucose status using anthropometric measures for diagnosing type 2 diabetes. IEEE J Biomed Health Inform 2014;18(2):555-61.

45. Marateb HR, Mansourian M, Faghihimani E, Amini M, Farina D. A hybrid intelligent system for diagnosing microalbuminuria in type 2 diabetes patients without having to measure urinary albumin. Comput Biol Med 2014;45:34-42.

46. Singh K, Singh VK, Agrawal NK, Gupta SK, Singh K.
Association of Toll-like receptor 4 polymorphisms with diabetic foot ulcers and application of artificial neural network in DFU risk assessment in type 2 diabetes patients. Biomed Res Int 2013;2013:318686.

47. Leung RK, Wang Y, Ma RC, Luk AO, Lam V, Ng M, et al. Using a multi-staged strategy based on machine learning and mathematical modeling to predict genotypephenotype risk patterns in diabetic kidney disease: a prospective case-control cohort analysis. BMC Nephrol 2013;14:162.

48. Wang C, Li L, Wang L, Ping Z, Flory MT, Wang G, et al. Evaluating the risk of type 2 diabetes mellitus using artificial neural network: an effective classification approach. Diabetes Res Clin Pract 2013;100(1):111-8.

49. Morteza A, Nakhjavani M, Asgarani F, Carvalho FL, Karimi R, Esteghamati A. Inconsistency in albuminuria predictors in type 2 diabetes: a comparison between neural network and conditional logistic regression. Transl Res 2013;161(5):397-405.

50. Mani S, Chen Y, Elasy T, Clayton W, Denny J. Type 2 diabetes risk forecasting from EMR data using machine learning. AMIA Annu Symp Proc 2012;2012:606-15.

51. Chikh MA, Saidi M, Settouti N. Diagnosis of diabetes diseases using an Artificial Immune Recognition System2 (AIRS2) with fuzzy K-nearest neighbor. J Med Syst 2012;36(5):2721-9.

52. Choubey DK, Paul S, Dhandhenia VK. Rule based diagnosis system for diabetes. An International Journal of Medical Sciences 2017;28(12):5196-208.

53. Zheng T, Xie W, Xu L, He X, Zhang Y, You M, et al. A machine learning-based framework to identify type 2 diabetes through electronic health records. Int J Med Inform 2017;97:120-7.

54. Agarwal V, Podchiyska T, Banda JM, Goel V, Leung TI, Minty EP, et al. Learning statistical models of phenotypes using noisy labeled training data. J Am Med Inform Assoc 2016;23(6):1166-73.

55. Jelinek HF, Stranieri A, Yatsko A, Venkatraman S. Data analytics identify glycated haemoglobin co-markers for type 2 diabetes mellitus diagnosis. Comput Biol Med 2016;75:90-7.

56. Yu CS, Liu CS, Chen RS, Lin CW. Artificial neural networks for estimating glomerular filtration rate by urinary dipstick for type 2 diabetic patients. Biomed Eng (Singapore) 2016;28(03):1650016.

57. Luo G. Automatically explaining machine learning prediction results: a demonstration on type 2 diabetes risk prediction. Health Inf Sci Syst 2016;4:2. 
58. Farran B, Channanath AM, Behbehani K, Thanaraj TA. Predictive models to assess risk of type 2 diabetes, hypertension and comorbidity: machine-learning algorithms and validation using national health data from Kuwait: a cohort study. BMJ Open 2013;3(5):e002457.

59. Meza-Palacios R, Aguilar-Lasserre AA, Urena-Bogarín EL, Vazquez-Rodriguez CF, Posada-Gomez R, TrujilloMata A. Development of a fuzzy expert system for the nephropathy control assessment in patients with type 2 diabetes mellitus. Expert Syst Appl 2017;72:335-43.

60. Sayadi M, Zibaeenezhad M, Taghi Ayatollahi SM. Simple prediction of type 2 diabetes mellitus via decision tree modeling. Int Cardiovasc Res J 2017;11(2):71-6.

61. Lee Y, Ragguett RM, Mansur RB, Boutilier JJ, Rosenblat JD, Trevizol A, et al. Applications of machine learning algorithms to predict therapeutic outcomes in depression: a meta-analysis and systematic review. J Affect Disord 2018;241:519-32.

62. Sisodia D, Sisodia DS. Prediction of diabetes using classification algorithms. Procedia Comput Sci 2018;132:1578-85.

63. Cruz JA, Wishart DS. Applications of machine learning in cancer prediction and prognosis. Cancer Inform 2007;2:59-77.

64. Du Y, Fu Z, Calhoun VD. Classification and prediction of brain disorders using functional connectivity: promising but challenging. Front Neurosc 2018;12:525.

65. Kalhori SR, Zeng XJ. Evaluation and comparison of different machine learning methods to predict outcome of tuberculosis treatment course. J Intell Learn Syst Appl 2013;5:184-93.

66. King RD, Feng C, Sutherland A. Statlog: comparison of classification algorithms on large real-world problems. Appl Artif Intell 1995;9(3):289-333.
67. Abdar M, Kalhori SR, Sutikno T, Subroto IM, Arji G. Comparing performance of data mining algorithms in prediction heart diseases. Int J Electr Comput Eng 2015;5(6):1569-76.

68. Kang S. Personalized prediction of drug efficacy for diabetes treatment via patient-level sequential modeling with neural networks. Artif Intell Med 2018;85:1-6.

69. Javad OM, Agboola S, Jethwani K, Zeid I, Kamarthi S. Reinforcement learning algorithm for blood glucose control in diabetic patients. Proceedings of ASME 2015 International Mechanical Engineering Congress and Exposition; 2015 Nov 13-19; Houston, TX.

70. Aniba MR, Siguenza S, Friedrich A, Plewniak F, Poch O, Marchler-Bauer A, et al. Knowledge-based expert systems and a proof-of-concept case study for multiple sequence alignment construction and analysis. Brief Bioinform 2009;10(1):11-23.

71. Cresswell K, Cunningham-Burley S, Sheikh A. Health care robotics: qualitative exploration of key challenges and future directions. J Med Internet Res 2018;20(7):e10410.

72. Ettore E, Wyckaert E, David R, Robert P, Guerin O, Prate F. Robotics and improvement of the quality of geriatric care. Soins Gerontol 2016;21(121):15-7.

73. Yeh CC, Spaggiari M, Tzvetanov I, Oberholzer J. Robotic pancreas transplantation in a type 1 diabetic patient with morbid obesity: a case report. Medicine (Baltimore) 2017;96(6):e5847.

74. Oberholzer J, Tzvetanov I, Mele A, Benedetti E. Laparoscopic and robotic donor pancreatectomy for living donor pancreas and pancreas-kidney transplantation. J Hepatobiliary Pancreat Sci 2010;17(2):97-100.

75. Management of type 1 diabetes in children is boosted by robots. Nurs Child Young People 2016;28(7):12. 